\title{
Next generation X-ray detectors for in-house XRD
}

\author{
Takeyoshi Taguchi ${ }^{\text {a) }}$ \\ Rigaku Corporation, 3-9-12 Matsubara-cho, Akishima-shi, Tokyo, Japan \\ Christian Brönnimann \\ DECTRIS Ltd., 5232 Villigen PSI, Switzerland and Paul Scherrer Institut, 5232 Villigen PSI, Switzerland \\ Eric F. Eikenberry \\ DECTRIS Ltd., 5232 Villigen PSI, Switzerland
}

(Received 4 September 2007; accepted 1 April 2008)

\begin{abstract}
A novel type X-ray detector, called PILATUS, has been developed at the Paul Scherrer Institut in Switzerland during the last decade. PILATUS detectors are two-dimensional hybrid pixel array detectors, which operate in single-photon counting mode. PILATUS detectors feature a very wide dynamic range $(1: 1000000)$, very short readout time $(<3.0 \mathrm{~ms})$, no readout noise, and very high counting rate $\left(>2 \times 10^{6}\right.$ counts $/ \mathrm{s} /$ pixel). In addition, a lower energy threshold can be set in order to suppress fluorescence background from the sample, thus a very good signal-to-noise ratio is achieved. The combination of these features for area detectors is unique and thus the PILATUS detectors are considered to be the next generation X-ray detectors. The basic building block of all the detectors is the PILATUS module having an active area of $83.8 \times 33.5 \mathrm{~mm}^{2}$. The PILATUS $100 \mathrm{~K}$ is a complete detector system with one module. PILATUS detector systems can have other configurations, including large area systems consisting of 20 to 60 modules that can cover up to an area of $431 \times 448 \mathrm{~mm}^{2}$. Such large systems are mainly used for macromolecular structure determination, such as protein crystallography and small angle X-ray scattering. The PILATUS $100 \mathrm{~K}$ detector can be easily adapted to many systems; the single-module detector is integrated to an in-house X-ray diffraction (XRD) system. Examples of XRD measurements with the PILATUS 100K detector are given. (C) 2008 International Centre for Diffraction Data.
\end{abstract}

[DOI: $10.1154 / 1.2912455]$

Key words: hybrid-pixel X-ray detector, radiation hard design, dynamic range, readout time, signal-to-noise ratio

\section{INTRODUCTION}

In order to take an X-ray image, various types of area detectors are developed and used. These include X-ray film, imaging plate, charge coupled device (CCD), multi-wire proportional counter, and flat panel. All of these detectors have good features as well as drawbacks. Analogue X-ray film has been used to take X-ray images since X-rays were discovered by Wilhelm Conrad Röentgen in 1895, and it is still widely used in various fields. The X-ray film is inexpensive and easy to obtain in large areas, but the film requires development and printing processes that are the same for conventional optical film, and the obtained image has to be digitized in order to analyze the intensity data using a computer. An imaging plate (IP) can be described as a substitution of the analogue film. IP does not require a dark room for image readout, but it takes about $1 \mathrm{~min}$ to read a $300 \times 300 \mathrm{~mm}^{2}$ image. A CCD detector is an order of magnitude faster than IP. However, the read-out time is in the order of seconds, typically a few seconds for $2 k \times 2 k$ format, and the X-ray source must be blocked by a mechanical shutter during the readout process.

All integrating area detectors are suffering from different sources of noise. The CCD detector has dark current and readout noise and therefore needs to be cooled to reduce the

\footnotetext{
${ }^{\text {a) }}$ Author to whom correspondence should be addressed. Electronic mail: takey@ rigaku.co.jp
}

dark current for long-time exposures. The CCD detector could be used for direct X-ray detection. However, the typical thickness of a few tens of microns is not sufficient enough to absorb $\mathrm{X}$-rays. A fluorescence screen, such as $\mathrm{GdO}_{x}$ film, is usually used to convert X-rays into visible light. The conversion efficiency of such screens is typically $40 \%$ to $60 \%$ and some portion of the incoming X-rays is lost.

A multi-wire proportional counter (MWPC) has the capability of single photon counting, and it can improve peak to background ratios significantly. The MWPC with a large detection area can easily be manufactured, and it has been widely used in many laboratories. The only problem is the counting rate. Slow ion transfer speed is a main cause of the counting rate limitation. The conventional MWPC has a global counting rate limitation of below $10^{5}$ counts/s.

A solid state area detector with a high counting rate, low background noise, and single photon counting capability is a long-time dream for many X-ray scientists. A long-time investigation and utilization of cutting edge technology at the Paul Scherrer Institut (PSI) makes this dream come true (Brönnimann et al., 1998, 2006).

\section{PILATUS DETECTOR}

The name PILATUS is an abbreviation for Pixel Apparatus for the SLS (Swiss Light Source). Currently, PILATUS has three variations, namely $6 \mathrm{M}, 2 \mathrm{M}$, and $100 \mathrm{~K}$. The PILATUS $6 \mathrm{M}$ covers an area of $424 \times 435 \mathrm{~mm}^{2}$, and is 


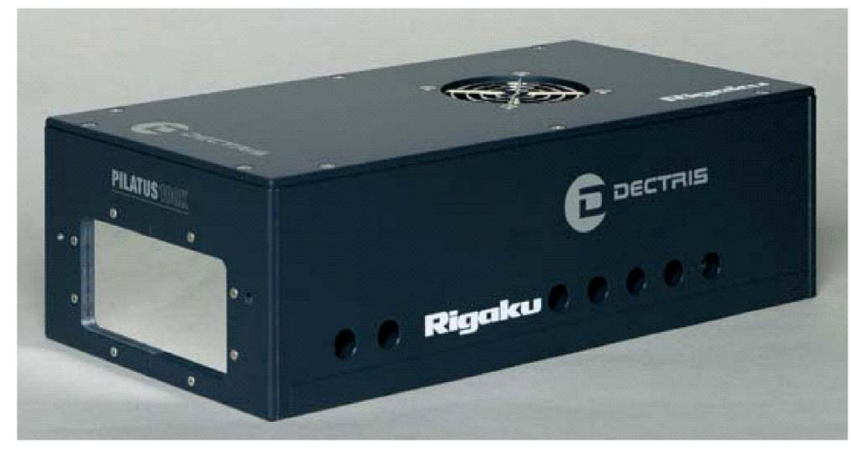

Figure 1. PILATUS 100K.

dedicated for protein crystallography. The PILATUS $2 \mathrm{M}$ is slightly smaller $\left(252 \times 268 \mathrm{~mm}^{2}\right)$ and designed for small angle X-ray scattering (SAXS). The PILATUS 100K (Figure 1) consists of one PILATUS II single module with detective area of $83.8 .5 \mathrm{~mm}^{2}$. For many experiments, a detector with this size is quite sufficient. With its outside dimensions of $256 \times 140 \times 85 \mathrm{~mm}^{3}$ and a weight of $4 \mathrm{~kg}$, it can be integrated easily into an in-house laboratory $\mathrm{X}$-ray apparatus.

\section{A. History}

The development of large area hybrid pixel detector started at the Paul Scherrer Institute in 1997. This detector was aimed to be used for protein crystallography. The specification of this detector was discussed thoroughly, and single-photon counting capability and fast data readout feature were desired. The single-photon counting capability reduces background noises, and thus data quality is improved. The fast readout feature reduces damage of protein crystals. In 2001, the first generation of PILATUS chip using DMILL's $0.8 \mu$ process was established. Then the first large area single photon counting pixel detector, the PILATUS 1M detector, was constructed and used to collect single crystal data in 2003. The second generation PILATUS chip (PILATUS II chip) was submitted in 2004 and showed greatly improved performance. Table I shows the specifications of the PILATUS I and II chips. The PILATUS 6M detector is based on this chip and was constructed in 2006. In parallel, the developers founded DECTRIS Ltd., a spin-off company of PSI in order to commercialize the PILATUS detector technology. With the PILATUS 6M detector, regular user operation was started at the SLS in the spring of 2007.

TABLE I. Properties of PILATUS I and II chips (Kraft, 2005).

\begin{tabular}{lcc}
\hline \hline Properties & PILATUS I & PILATUS II \\
\hline Technology & DMILL & UMC 0.25 \\
Active area & $10.1 \times 17.7 \mathrm{~mm}^{2}$ & $10.45 \times 17.54 \mathrm{~mm}^{2}$ \\
Number of pixels & $44 \times 78=3432$ & $60 \times 97=5820$ \\
Pixel size & $217 \times 217 \mu \mathrm{m}^{2}$ & $172 \times 172 \mu \mathrm{m}^{2}$ \\
Counter (pixel) & $15 \mathrm{bit}$ & $20 \mathrm{bit}$ \\
Count rate & $<10 \mathrm{kHz} /$ pixel & Up to $3 \mathrm{MHz} /$ pixel \\
\hline \hline
\end{tabular}

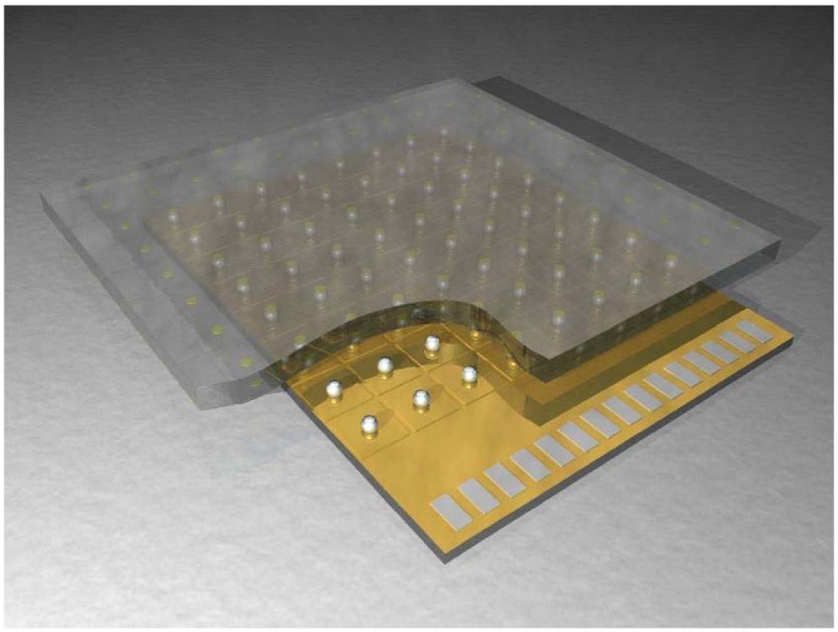

Figure 2. (Color online) Hybrid detector scheme.

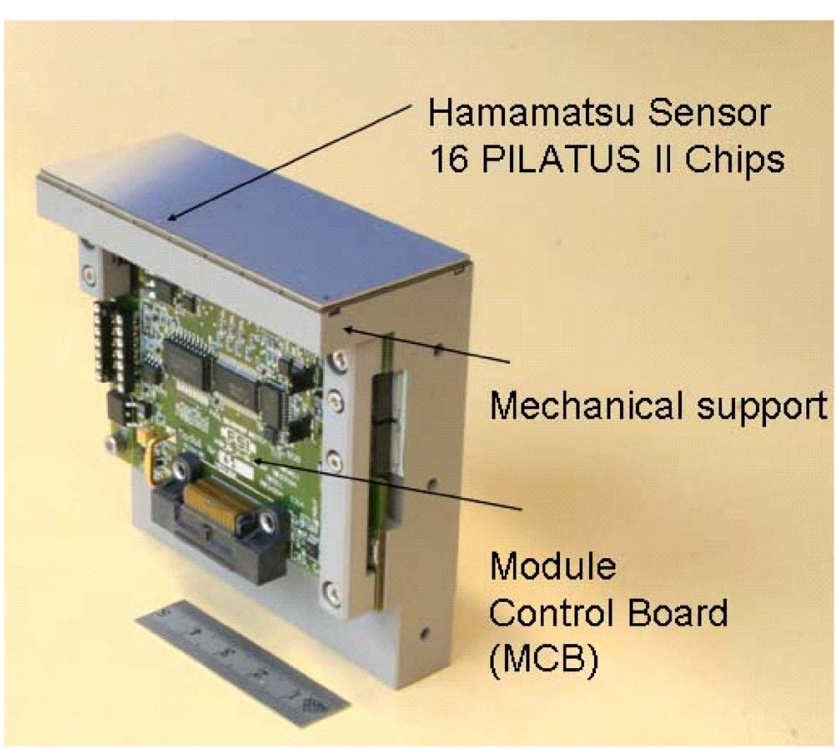

Figure 3. (Color online) PILATUS II module.

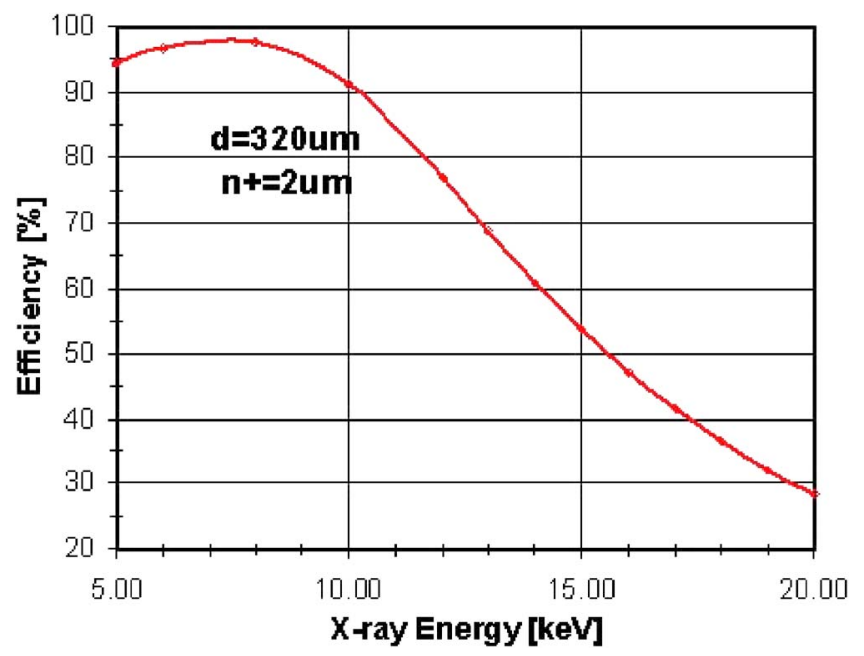

Figure 4. (Color online) PILATUS 100K sensor (detective quantum efficiency). 


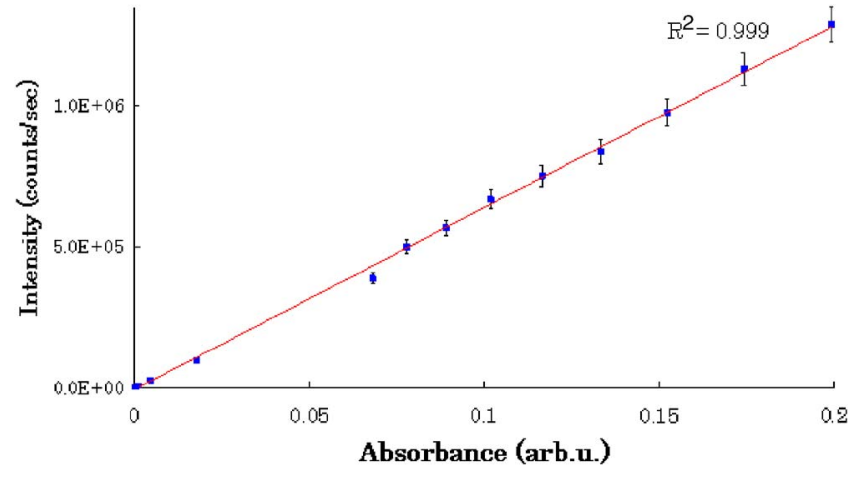

Figure 5. (Color online) Counting rate of single pixel.

\section{B. Technology}

The detector consists of a two-dimensional array of pndiode, usually processed in high resistivity silicon, connected to the readout Application Specific Integrated Circuit (ASIC), which is designed with the latest complementary metal-oxide semiconductor technology. These two parts are connected by small metal balls (bump-bonding). In principle, the sensor can be chosen out of a variety of materials. Hence, this type of detector is often called "hybrid detector" (Figure 2). PILATUS II single module consists of $16(2 \times 8)$ PILATUS II readout ASICs which are bump-bonded to a $320-\mu \mathrm{m}$-thick monolithic silicon sensor. The assembly is connected to a mechanical support carrying the module control board (Figure 3). After assembling, the detector is calibrated by exposing it to a homogeneous field of X-rays, and the trim bit of each pixel is adjusted in order to have a uniform threshold (Brönnimann et al., 2000).

For X-ray energies above $8 \mathrm{keV}, \mathrm{X}$-rays penetrate the sensor and a certain dose is deposited in the readout chip. Therefore a radiation hard design is applied and the PILATUS II chip can tolerate more than 30 Mrad dose.

A fully depleted $320-\mu \mathrm{m}$-thick silicon sensor absorbs $99 \%$ of incident $\mathrm{X}$-ray at $8 \mathrm{keV}$ and $40 \%$ at $17 \mathrm{keV}$ (Figure 4). Since most in-house $X$-ray diffraction experiments are carried out with $\mathrm{Cu} K \alpha \mathrm{X}$-rays $(8 \mathrm{keV})$, there is no need to make the silicon sensor thicker or to replace it with other materials such as GaAs or CdTe. For some experiments using Mo $K \alpha \mathrm{X}$-rays $(17 \mathrm{keV})$, the efficiency goes down to less than $40 \%$, but it still provides sufficient performance. Thicker silicon sensors are possible, however it is difficult to make large monolithic sensors with GaAs or CdTe.

\section{Performance}

Since PILATUS is a single-photon counting digital detector, it is not suffering from dark current or readout noise. For setting the threshold level correctly, PILATUS covered with a lid only counts cosmic background for minutes-long exposure. High counting rate capability was tested by putting
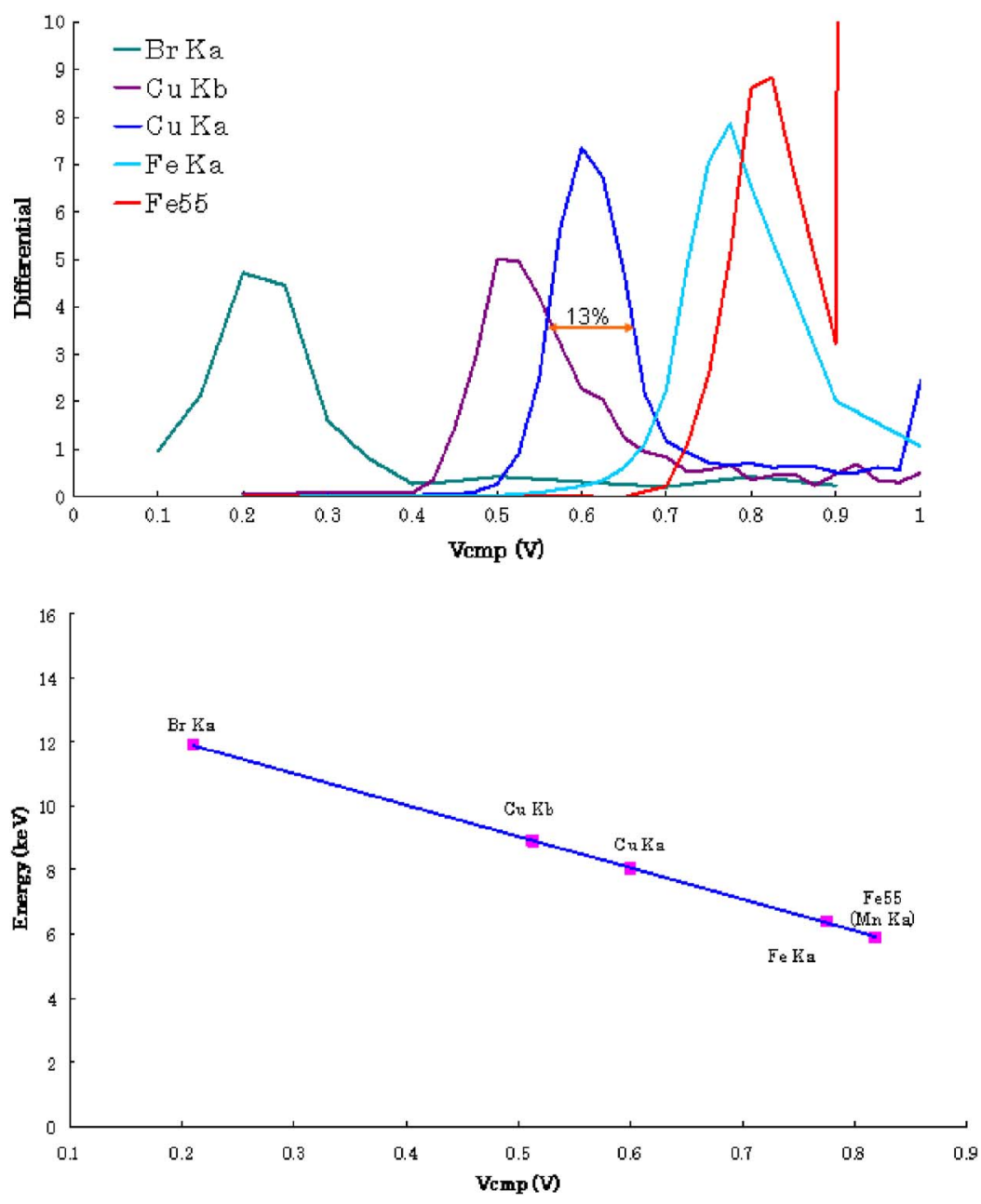

Figure 6. (Color online) Threshold scan result (top: peak width; bottom: energy linearity). 

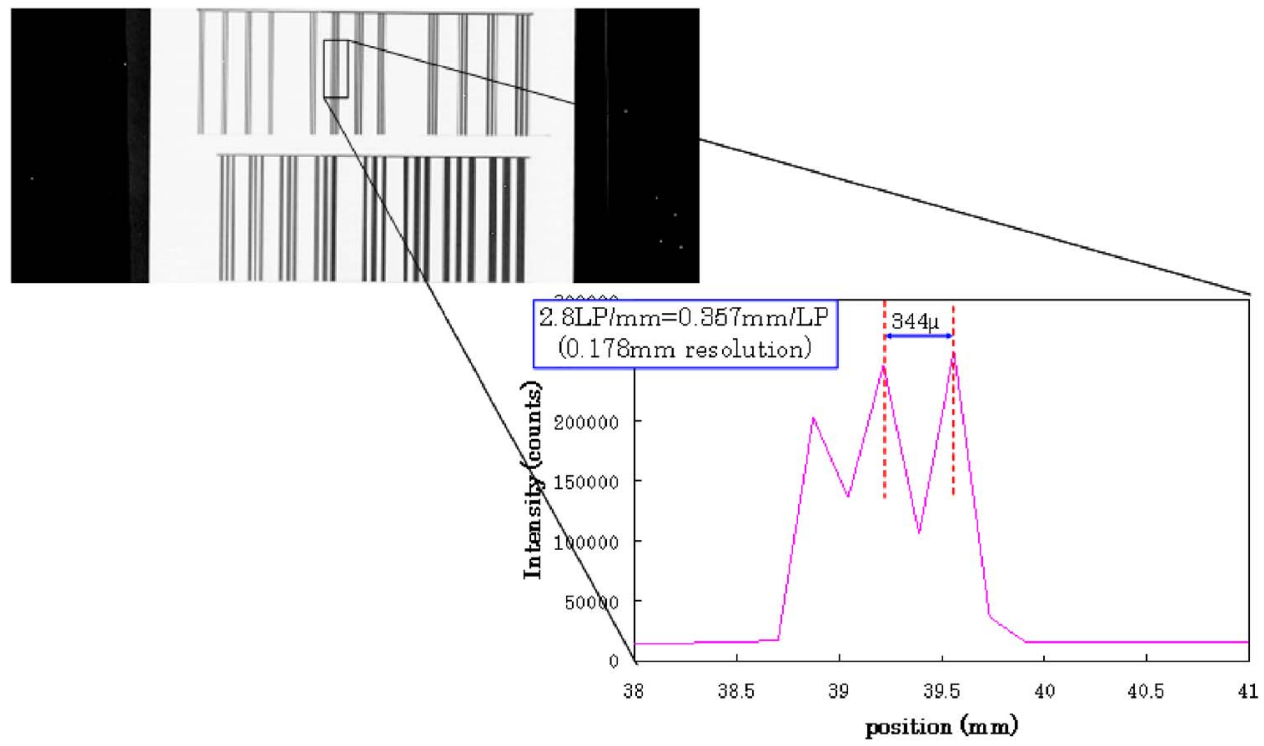

Figure 7. (Color online) Spatial resolution test result.

thin aluminum foils in front of the detector. In Figure 5, the number of photons counted in a second is plotted against the thickness of the absorber. Counting rates beyond $10^{6} \mathrm{cps} /$ pixel were confirmed with a laboratory X-ray source. However, since this is a counting detector, count-rate corrections need to be applied, for rates above $\sim 100 \mathrm{kcts} / \mathrm{s} /$ pixel. In order to cover different experimental needs, there are three settings of the pixel amplifiers. For standard settings, the dead time measured is set to $\sim 200 \mathrm{~ns}$. For X-rays with energies below $5 \mathrm{keV}$, high-gain setting may be used and dead time of $300 \mathrm{~ns}$ is applied. The lowgain mode can be chosen for very high counting rate measurement with dead time of $129 \mathrm{~ns}$.

PILATUS detectors have a lower threshold level so that it can suppress fluorescence background. X-rays of various energies $(5.9,6.4,8,8.9$, and $11.9 \mathrm{keV})$ were irradiated, and output intensity was measured by scanning the threshold level by changing the value of Vcmp. As a result, it is ob-

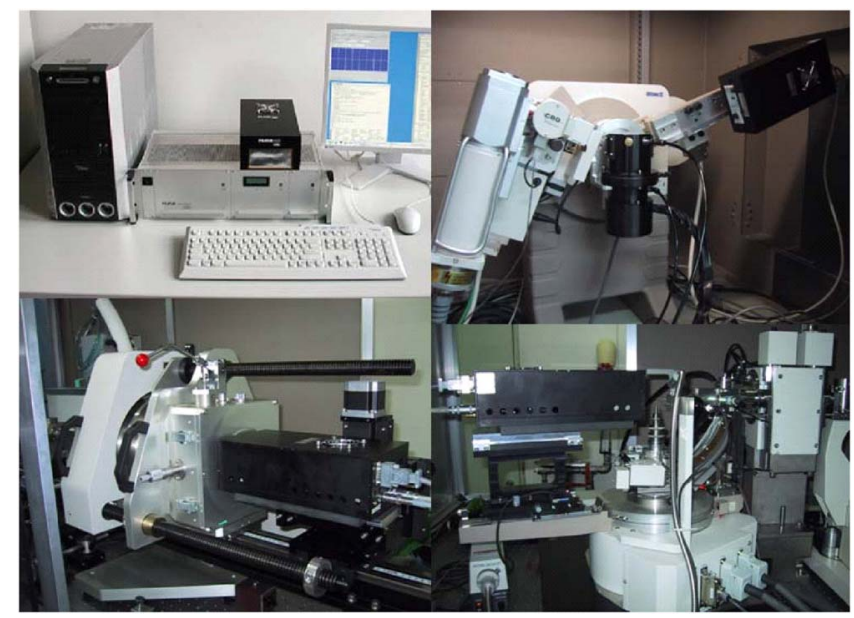

Figure 8. (Color online) Combination of PILATUS 100K (top left: PILATUS 100K system; top right: PILATUS 100K with XRD-DSC II; bottom left: PILATUS 100K with Nano-Viewer; bottom right: PILATUS 100K with AFC-10). served that the FWHM of $\mathrm{Cu} K \alpha \mathrm{X}$-rays is about $13 \%$ $(130 \mathrm{mV} / 90 \mathrm{mV} / \mathrm{keV} / 2.35)$ and the $\mathrm{Vcmp}$ value is linear to input energy (Figure 6).

Because PILATUS is a solid state pixel detector, it is not expected to be affected by parallax like a gas counter. An $\mathrm{X}$-ray test chart with various line spacings was put in front of the detector and irradiated by a micro focus X-ray source with $10 \mu \mathrm{m}$ focal spot size from a distance of $500 \mathrm{~mm}$. The 2.8 line pair/mm, or $0.357 \mathrm{~mm} /$ line pair, feature was resolved with about 50\% modulation (Figure 7). This indicates the spatial resolution of this detector is about $178 \mu \mathrm{m}$, which is equivalent to the pixel size. Thus the point spread function of this detector is the same as the pixel size.
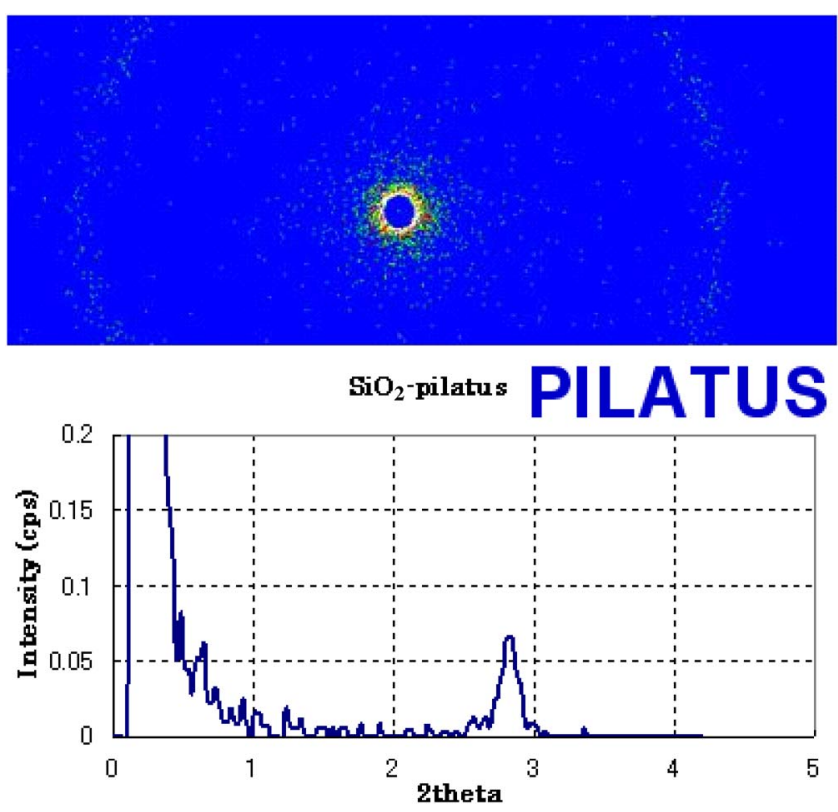

Figure 9. (Color online) SAXS pattern (top) and the annual integration (bottom). 


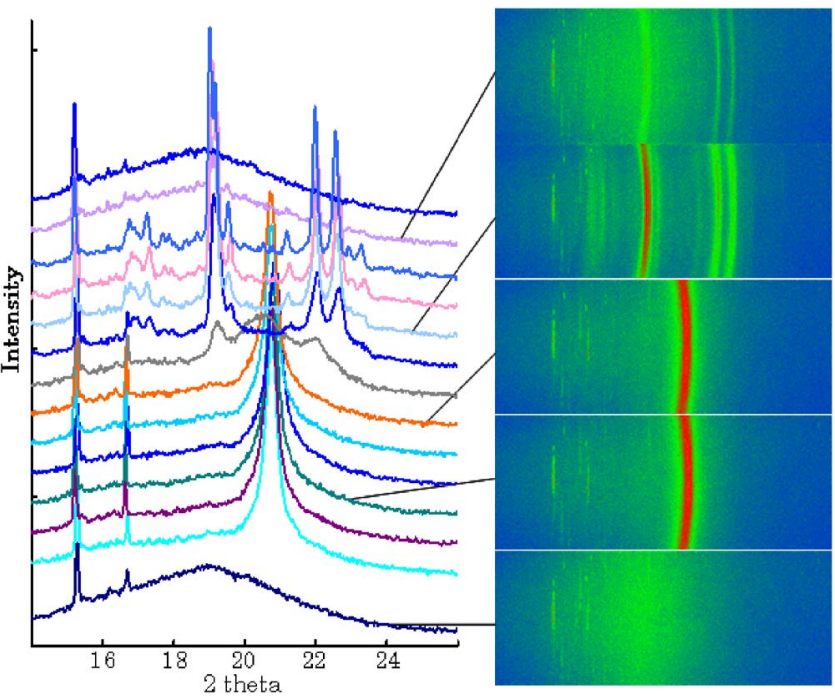

Figure 10. (Color online) Phase transition of Tripalmitin.

\section{Applications}

A PILATUS $100 \mathrm{~K}$ detector system was combined with various in-house XRD systems (Figure 8) and tested. The integration to in-house systems was surprisingly easy and the detector started up instantaneously.

One of the most attractive features of the PILATUS $100 \mathrm{~K}$ is its very low background noise and very high counting rate. It is rare to find these two features combined in conventional X-ray detectors. These two features are particularly good for SAXS measurements: Figure 9 shows a SAXS data of meso-porous silica $\left(\mathrm{SiO}_{2}\right)$ measured with a PILATUS $100 \mathrm{~K}$ detector. The exposure time is $1 \mathrm{~s}$ and the intensity of a $60^{\circ}$ to $120^{\circ}$ arc is accumulated and plotted. The background for the whole data range is almost zero, and the weak scattering peak at $2.8^{\circ} 2 \theta$ is clearly visible. According to this data, signals less than 0.05 counts/s can be observed. Thus very faint scattering can be observed by using the PILATUS $100 \mathrm{~K}$ detector. One of the other advantages of using this detector for SAXS experiments is the parallax-free measurement with good point spread function described below. Using conventional area detectors, such as IP or CCDs, the exposure time needs to be longer and a higher background is usually observed.
One of the other key features of PILATUS is the fast framing rate of $300 \mathrm{~Hz}$. It can collect data without shutter control, minimizing the dead time and it can thus easily be used for dynamical phase transition study, for example experiments as carried out using a small area detector (Taguchi, 2006). The phase transition process of Tripalmitin (neutral lipid) was observed using the XRD and DSC (differential scanning calorimetry) combined apparatus with PILATUS $100 \mathrm{~K}$. The sample was heated up to $85^{\circ} \mathrm{C}$ and phases were observed in cooling stage (Figure 10). The PILATUS 100K can cover about $17^{\circ} 2 \theta$ with angular resolution of $0.0346^{\circ}$ at the distance of $285 \mathrm{~mm}$ from the specimen.

\section{SUMMARY}

The PILATUS 100K detector system is combined with in-house XRD systems. It provides new, quality data compared with conventional detectors and will open new frontiers in the field of X-ray diffraction for in-house XRD users as well as synchrotron radiation users.

\section{ACKNOWLEDGMENTS}

The authors thank P. Salficky of DECTRIS Ltd. for his technical help. T. Taguchi thanks Dr. Iwasaki Yoshio of Rigaku Corporation for the SAXS experiment and interpretation of the results.

Brönnimann, C., Baur, R., Horisberger, R., and Schulze, C. (1998). "Pixel detector for the protein crystallography (PX) beamline," SLS Annex report about the pixel detector development, Swiss Light Source, Paul Scherrer Institut, Villigen PSI, Switzerland, 〈http://pilatus.web.psi.ch/ DATA/PDF/Jb98_pix.pdf $>$.

Brönnimann, C., Florin, S., Linder, M., Schmitt, B., and Schulze-Briese, C. (2000). "Synchrotron beam test with a photon-counting pixel detector," J. Synchrotron Radiat. 7, 301-306.

Brönnimann, C., Eikenberry, E. F., Henrich, B., Horisberger, R., Huelsen, G., Pohl, E., Schmitt, B., Schulze-Briese, C., Suzuki, M., Tomizaki, T., Toyokawa, H., and Wagner, A. (2006). "The PILATUS 1M detector," J. Synchrotron Radiat. 13, 120-130.

Kraft, P. (2005). "Characterization of the readout chip for the PILATUS 6M detector," Diploma thesis, Institute for Particle Physics, Swiss Federal Institute of Technology (ETH Zürich), Zürich, Switzerland 〈http:// pilatus.web.psi.ch/DATA/PDF/Kraft_diploma.pdf $\rangle$.

Taguchi, T. (2006). "A new position sensitive area detector for high-speed and high-sensitivity X-ray diffraction analysis," Powder Diffr. 21, 97101. 